\title{
Enhanced sensitivity fibre optic long period grating temperature sensor
}

Sarfraz Khaliq, Stephen W. James* and Ralph P. Tatam

Optical Sensors Group

Centre for Photonics and Optical Engineering

School of Engineering

Cranfield University

Cranfield

Bedford

MK43 OAL

UK

$$
\begin{aligned}
& \text { Tel +44 (0) } 1234754623 \\
& \text { Fax +44 (0) } 1234750728
\end{aligned}
$$

Email s.w.james@cranfield.ac.uk

* Corresponding author 


\section{Abstract}

Surrounding a long period grating, fabricated in a standard optical fibre, with a material with a high thermo-optic coefficient enhances the temperature sensitivity of a long period grating. Temperature sensitivities as high as $19 \mathrm{~nm} /{ }^{\circ} \mathrm{C}$ are reported over a temperature range of $1.1^{\circ}$. The temperatures at which the LPG exhibits enhanced sensitivity may be tailored by a suitable choice of material.

Keywords: Fibre optics; Fibre optic sensors; Long period grating. 


\section{Introduction}

The fibre optic long period grating (LPG) offers a number of interesting properties for sensing applications. The characteristic attenuation bands in the transmission spectrum are sensitive to a range of measurands, particularly strain ${ }^{1}$, temperature ${ }^{1}$, bend radius ${ }^{2,3,4}$ and the external refractive index $(\mathrm{RI})^{5,6}$. Each attenuation band shows a different sensitivity to the measurands, offering the potential for the development of multi-parameter sensing systems ${ }^{7}$. The sensitivity to a particular measurand is determined by the composition of the fibre and its construction. Changing the chemical composition of the fibre allows the LPG to exhibit positive, negative or zero response to temperature and strain $^{8}$, while changing the structure of the fibre has been shown to allow enhancement of the temperature response ${ }^{9}$. In this paper we demonstrate a simple technique for forming a LPG with enhanced temperature sensitivity by surrounding the LPG with a material with a high thermo-optic coefficient, thus exploiting both the temperature and external RI sensitivity of the LPG. The operating temperature and measurement ranges are determined by the attenuation band that is monitored, and the refractive index and the thermo-optic coefficient of the surrounding material.

A LPG consists of a periodic modulation of the RI of the core of an optical fiber. The periodicity lies typically in the range $100 \mu \mathrm{m}$ to $1000 \mu \mathrm{m}$. The small grating wave vector promotes the coupling of light from the propagating core mode to co-propagating cladding modes. Since the cladding modes suffer from high attenuation, the transmission spectrum consists of a series of attenuation bands centered on wavelengths given by ${ }^{1}$

$$
\lambda_{i}=\left[n_{e f f}\left(\lambda_{i}\right)-n_{\text {clad }}^{i}\left(\lambda_{i}\right)\right] \Lambda
$$


where $\lambda_{\mathrm{i}}$ is the coupling wavelength, $\mathrm{n}_{\mathrm{eff}}$ is the effective index of the propagating cladding mode, $\mathrm{n}^{(i)}$ clad is the index of the ith cladding mode, and $\Lambda$ is the period of the LPG. The temperature sensitivity of the coupling wavelengths arises from the difference between the thermo-optic coefficients of the cladding and core, while the refractive index, RI, sensitivity arises from the dependence of the cladding mode's effective RI and upon the RI of the surrounding material.

A LPG of length $40 \mathrm{~mm}$, period $400 \mu \mathrm{m}$, fabricated in boron-germanium co-doped optical fiber (Fibercore PS750) with cut off wavelength $650 \mathrm{~nm}$ was submerged in a series of Cargille oils of different refractive indices. The transmission spectrum of the LPG surrounded by air is shown in Figure 1. Figure 2 shows the bulk external medium RI induced wavelength shift and the change in the minimum transmission value of the attenuation band corresponding to coupling to the $5^{\text {th }}$ order cladding mode. The figure shows that the wavelength response to RI occurs over a range of approximately 1.400 to 1.456 . The upper limit is imposed by the RI of the fibre's cladding. For a RI above this limit there is no wavelength response. The minimum transmission values of the attenuation bands show a response to refractive indices in the range 1.44 to 1.456 . At an external refractive index of 1.456 the attenuation bands disappear, to reappear for RIs $>1.460$, but with reduced minimum transmission. The use of Cargille oils to characterise the RI response of LPG has been reported previously ${ }^{5}$, however, the influence of the thermo-optic response of the oils has not been previously investigated in detail.

The LPG, when surrounded by air, shows a temperature sensitivity in the range of $-0.14 \mathrm{~nm} /{ }^{\circ} \mathrm{C}$ to $-0.34 \mathrm{~nm} /{ }^{\circ} \mathrm{C}$, dependant upon the attenuation band being monitored. This sensitivity is approximately 2 orders of magnitude larger than that of a fibre Bragg grating ${ }^{10}$. Previous reports of temperature sensitivity enhancement of LPGs have relied upon the use of novel cladding structures ${ }^{9}$, producing a sensitivity of $0.8 \mathrm{~nm} /{ }^{\circ} \mathrm{C}$, or upon etching the fibre to produce an ultra thin 
cladding and surrounding it with a liquid crystal material, resulting in a temperature sensitivity of $2.1 \mathrm{~nm} /{ }^{\circ} \mathrm{C}^{11}$. The technique reported here surrounds a LPG, fabricated in a standard optical fibre, with a liquid with a high thermo-optic coefficient. Temperature induced RI changes of the liquid then induce changes in the transmission spectrum of the LPG which, over a limited temperature range, results in enhanced temperature sensitivity.

The transmission spectrum was monitored by coupling the output from a white light source into the optical fiber containing the LPG (parameters as described previously), and coupling the output from the distal end of the fiber into a PC interfaced CCD spectrometer (Ocean Optics S2000). The spectrometer offers a measurement range of $500 \mathrm{~nm}$ to $1100 \mathrm{~nm}$, with a resolution of $0.3 \mathrm{~nm}$ and a minimum integration time of $1 \mathrm{~ms}$. The fibre was immersed in Cargille RI oil of RI 1.462. The temperature of oil was controlled via a thermo-electric cooler to $\pm 0.01^{\circ}$. The wavelength and minimum transmission value of each attenuation band was recorded as a function of temperature.

Figure 3(a) shows the shift of the central wavelengths of the attenuation bands as a function of temperature, while Figure 3(b) shows the change in minimum transmission value of the attenuation bands. As the RI of the oil is greater than that of the cladding, the attenuation bands are reduced in depth at room temperature, RT. The thermo-optic coefficient of the RI oil is negative, and thus increasing the temperature results in a reduction in RI. Once the index is less than or equal to that of the cladding of the fibre, the central wavelengths of the attenuation band respond to the changing RI. Figure 3 (a) shows that each attenuation band has a different temperature sensitivity, and that, over a limited range, very high temperature sensitivities are achieved (up to $19.2 \mathrm{~nm} /{ }^{\circ} \mathrm{C}$ over a temperature range of $1.1^{\circ} \mathrm{C}$ ). It is also interesting to note that the temperature range is different for each attenuation band. This is a result of the dispersion of the RI oil and of the cladding of the fibre. The depth of the attenuation band also demonstrates a large temperature sensitivity of up to 
$14.8 \% /{ }^{\circ} \mathrm{C}$ over a temperature range of $2.0^{\circ} \mathrm{C}$, and again, offers different operating ranges for the different attenuation bands.

If a different operating range is required, it is possible to choose a material with a different RI at room temperature, such that its RI lies within the sensitivity range of the LPG over a different range of temperatures. This is illustrated by the results shown in figure 4 , where the temperature responses of the attenuation band corresponding to coupling to the $5^{\text {th }}$ cladding mode surrounded by two different RI oils ( $\mathrm{n}=1.460, \mathrm{n}=1.462$ ) are compared, demonstrating the tuning of the operating temperature range by change of material.

The measurements reported were found to be reproducible and no hysteresis was observed during temperature cycling. The temperature sensitivity enhancement discussed here would be of use in temperature critical systems, for example in chemical and material processing, where optimum processing temperature range may be limited. In addition, the high temperature sensitivity is of interest for thermally tuned optical filters.

In summary, a method for enhancing the temperature sensitivity of a LPG has been discussed. The LPG, fabricated in standard optical fibre, is surrounded by a Cargille refractive index oil with a high thermo-optic coefficient. There is no requirement for novel coating materials or novel fibre structures. A sensor based upon this principal could be readily constructed using a tube filled with the appropriate oil. The tube would also provide support for the LPG, avoiding bending induced changes to the transmission spectrum ${ }^{2-4}$. The LPG exhibits a temperature sensitivity of $19.2 \mathrm{~nm} /{ }^{\circ} \mathrm{C}$ over a temperature range of $1.1^{\circ} \mathrm{C}$. Monitoring a number of the attenuation bands in the spectrum of a single LPG allows measurements to be performed over different temperature ranges. The operating temperature and the sensitivity are determined by the properties of the surrounding oil, 
allowing optimisation of the sensor for a given application. The results presented also show that great care must be taken when using LPG to measure the refractive index of materials that change with large thermo-optic coefficients.

The authors acknowledge support of the Engineering and Physical Sciences Research Council, U.K., GR/M87337/01, the Royal Society, U.K. and Dr. David. W. Coutts of Oxford lasers for supplying the amplitude mask used to fabricate the LPG. 


\section{Figure Captions}

Figure 1: Transmission spectrum of the LPG, used for the experiments, in air at room temperature, recorded following annealed at $120{ }^{\circ} \mathrm{C}$ for 3 hours.

Figure 2: $\quad$ Plot of (a) the wavelength shift (nm) and (b) the minimum transmission value of the attenuation bands against the RI of the material surrounding the LPG, for the central wavelength of the attenuation band corresponding to coupling to the $5^{\text {th }}$ cladding mode, att. band 5 . The lines through the data points are a guide to the eye.

Figure 3a: Plot of the wavelength shift in the central wavelengths of the 5 attenuation bands, att. bands 2-6, as a function of temperature. The wavelength shift is calculated with respect to the wavelength of the respective attenuation band in air at $25^{\circ} \mathrm{C}$. $\bullet$ att. band 2, * att. band 3, $\sigma$ att. band $4, \times$ att. band 5 and $\boldsymbol{\square}$ att. band 6

Figure 3b: Plot of the change in the minimum transmission value of the 5 attenuation bands, att. bands 2-6, as a function of temperature. The change in the minimum transmission value of the 5 attenuation bands is calculated w.r.t the minimum transmission value of the respective att. band in air at $25^{\circ} \mathrm{C}$. $\bullet$ att. band $2, *$ att. band 3, $\sigma$ att. band 4 , $\times$ att. band 5 and $\boldsymbol{m}$ att. band 6

Figure 4: Plot of the wavelength shift in central wavelengths in response to temperature for att. band 5, when surrounded by two recorded in air at $25^{\circ} \mathrm{C}$. att. band 5 in oil of RI 1.460 (at $25^{\circ} \mathrm{C}$ with a wavelength of $589.3 \mathrm{~nm}$ ), $\sigma$ att. band 5 in oil of RI 1.462 (at $25^{\circ} \mathrm{C}$ with a wavelength of $589.3 \mathrm{~nm}$ ) 


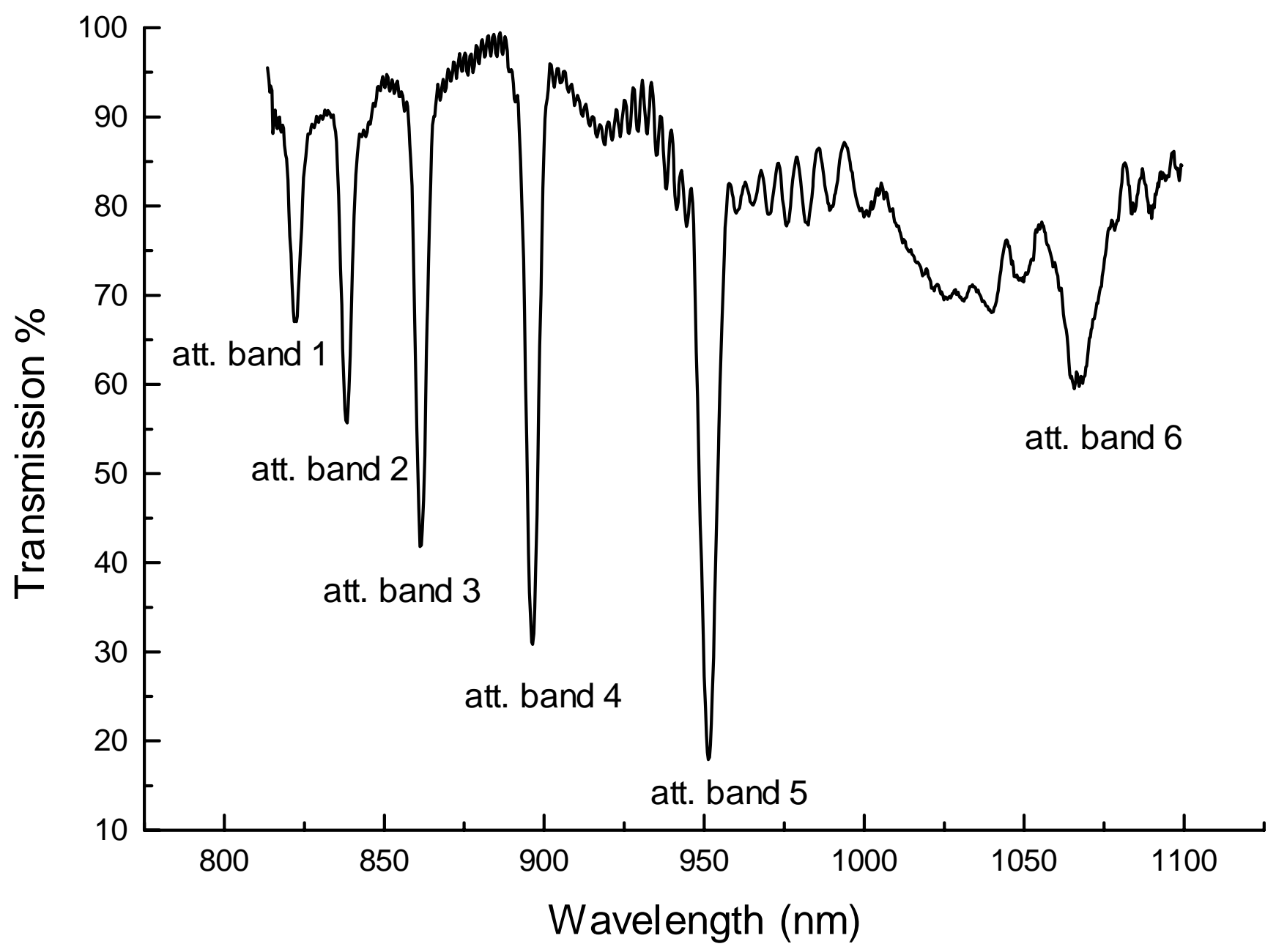

Figure 1. 

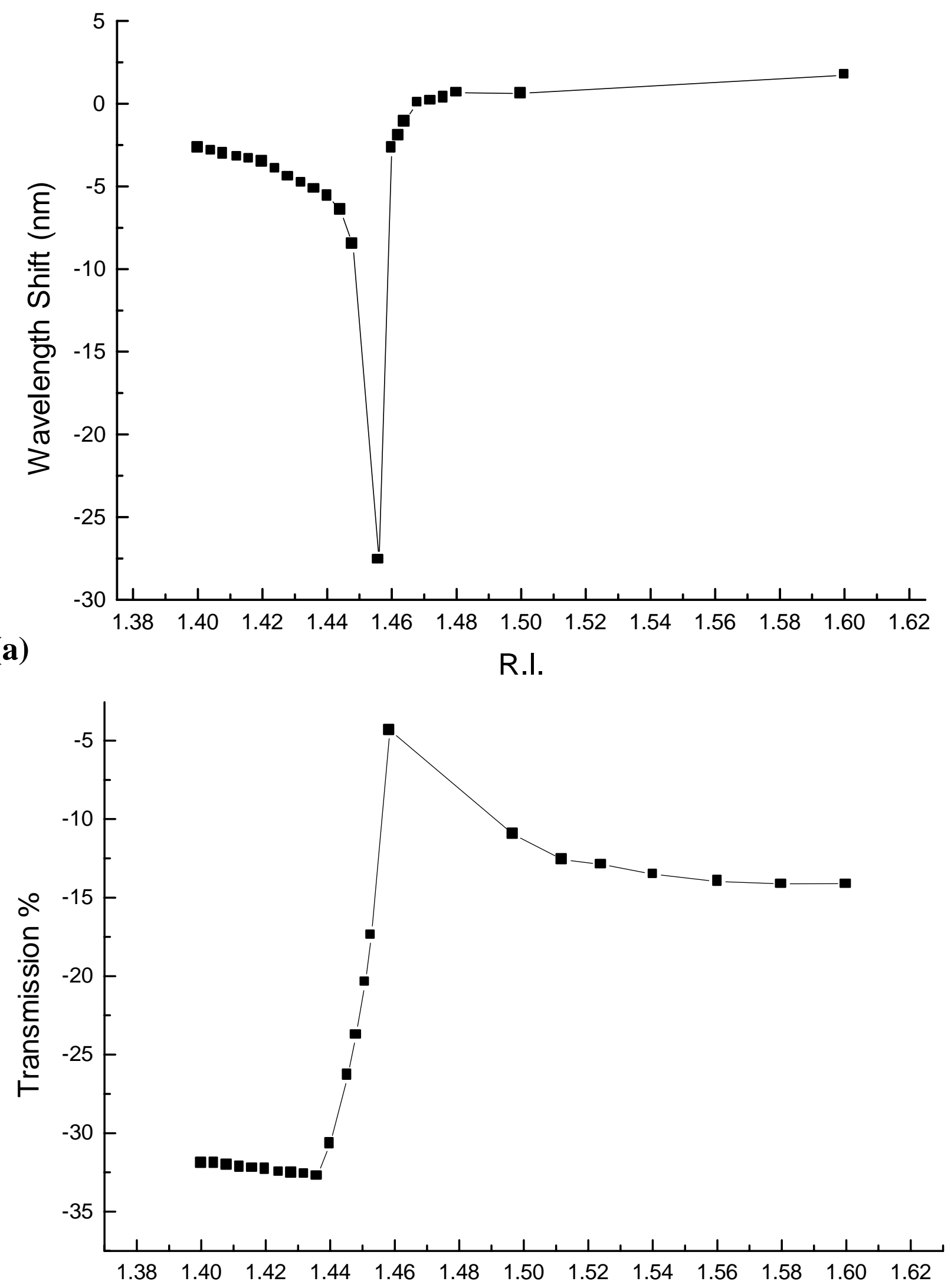

(b)

R.I.

Figure 2: 


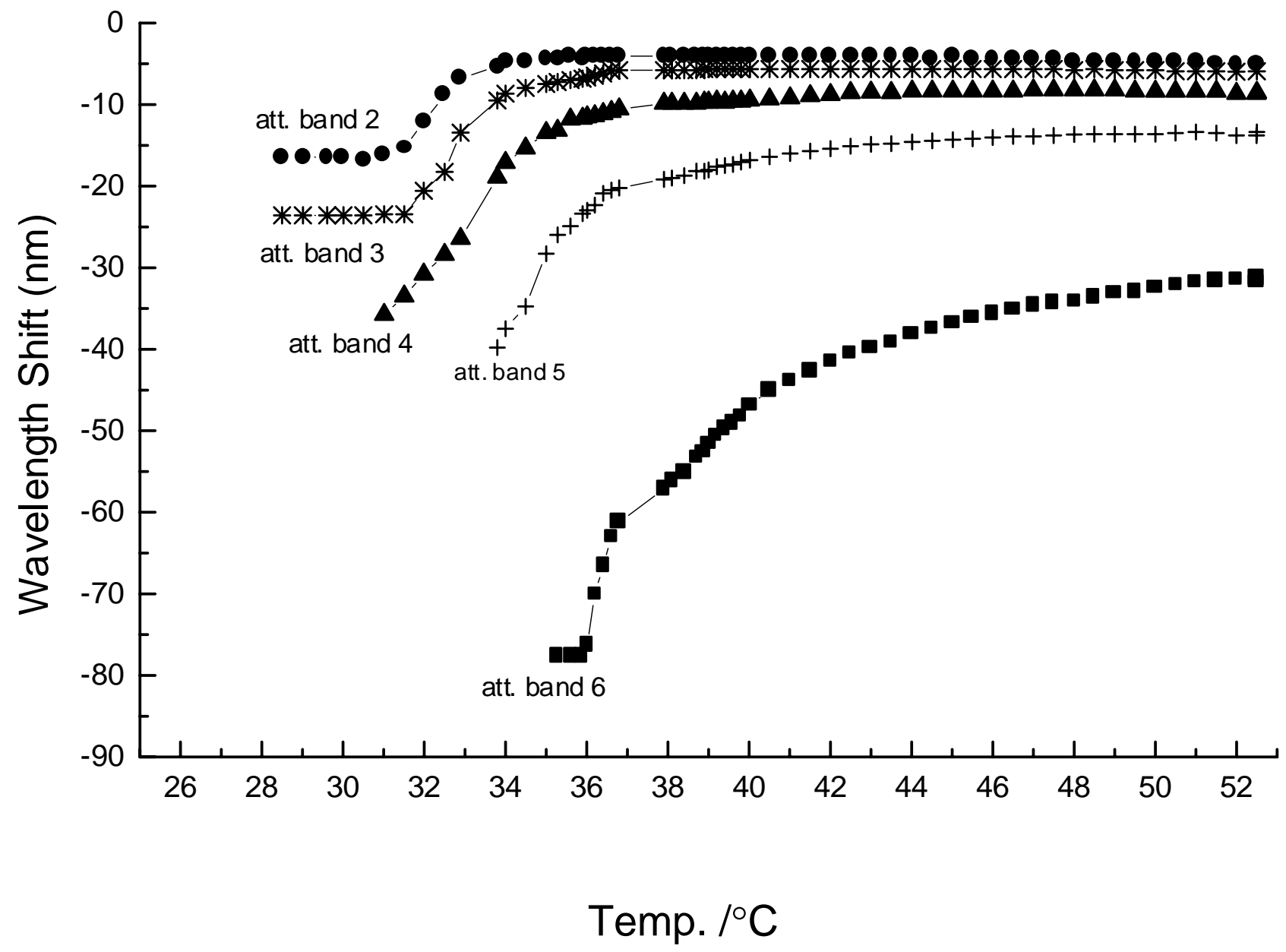

Figure 3a: 


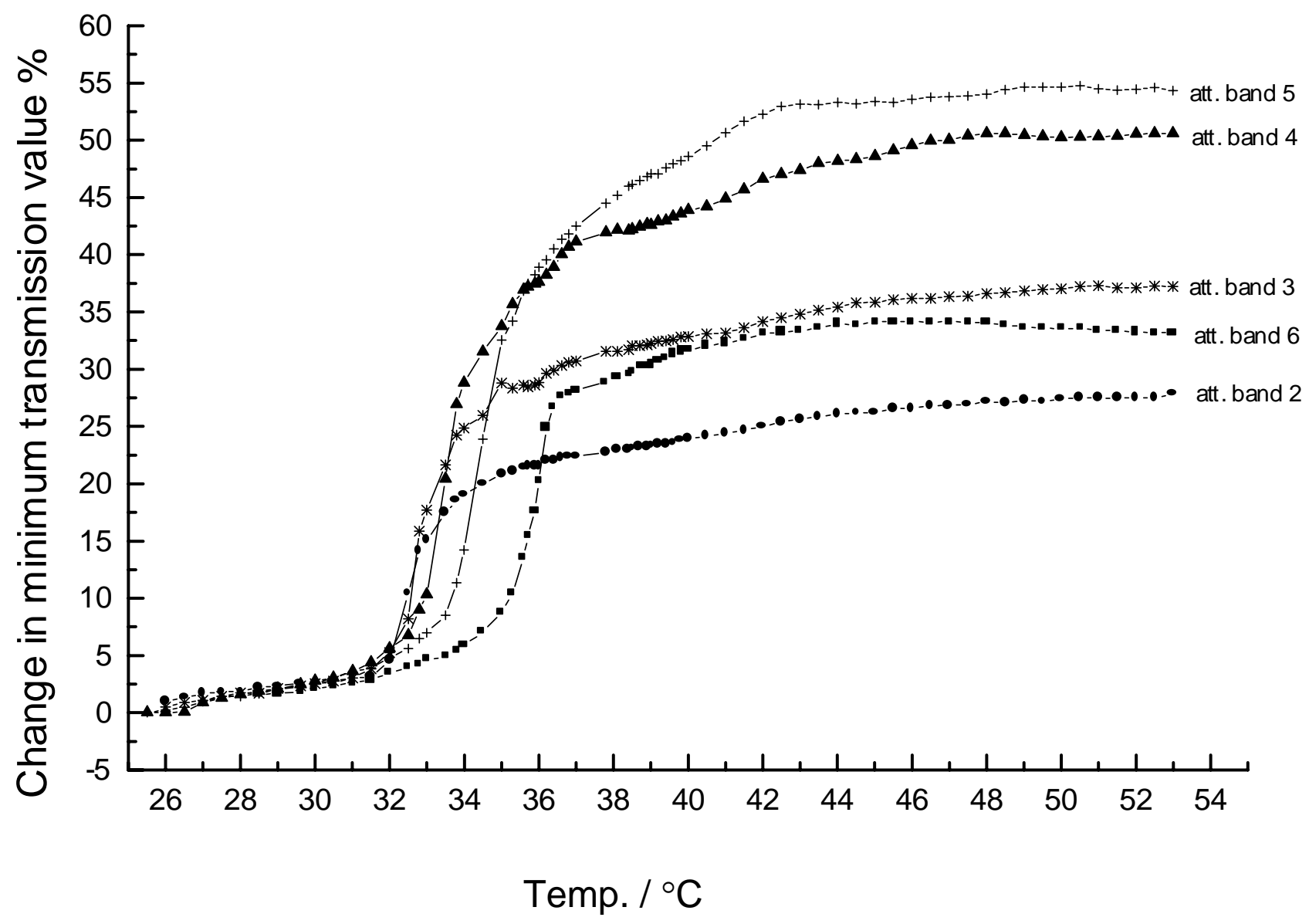

Figure 3b: 


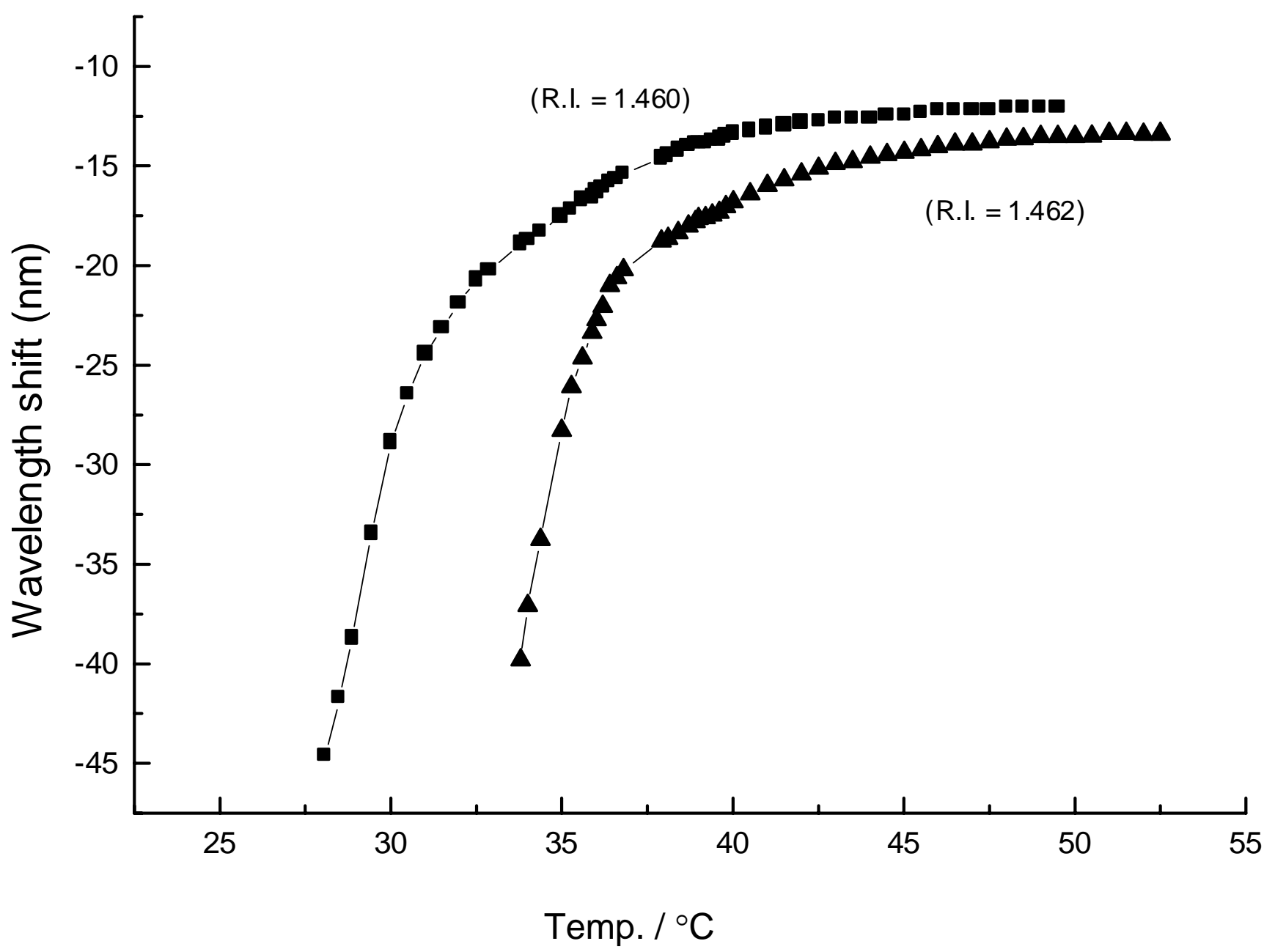

Figure 4: 


\section{References}

1. V. Bhatia, D.K. Cambell, D.Sherr, T.G. D’Alberto, N.A. Zabaronick, G.A. Ten Eyck, K.A. Murphy and R.O. Claus, Opt. Eng. 36, 1872 (1997).

2. $\quad$ C.C.Ye, S.W. James and R.P. Tatam, Opt. Lett. 25, 14, 1007 ( 2000).

3. H.J. Patrick, Electron. Lett. 36, 1763 (2000).

4. Y. Liu, L. Zhang, J.A.R. Williams and I. Bennion, IEEE - Photonic Technol. Lett. 12, 531 (2000).

5. H.J. Patrick, A.D. Kersey and F. Bucholtz, J. Lightwave Technol. 16, 1606 (1998).

6. S. Khaliq, S.W. James and R.P Tatam, Opt.Lett. 26, 1224 (2001).

7. V. Bhatia, Optics Express 4457 (1999)

8 V. Bhatia, D.K. Campbell, D. Sherr, T.G. D’Alberto, N.A. Zabaronick, G.A. Ten Eyck, K.A. Murphy and R.O. Claus. Opt.Eng. 36, 1872 (1997).

9 A.A.Abramov, A.Hale, R.S.Windeler and T.A.Strasser, Electron. Lett., , 35, 81 (1999)

10 S. Magne, S. Rougeault, M. Viela and P. Ferdinand, Appl.Opt. 36, 9437 (1997)

11 S. Yin, K-W Chung and X. Zhu, Opt.Comm. 188, 301 (2001). 